University of New Orleans

ScholarWorks@UNO

\title{
$5-2011$
}

\section{Blending Geospatial Technology and Traditional Ecological Knowledge to Enhance Restoration Decision-Support Processes in Coastal Louisiana}

\author{
Matthew B. Bethel \\ University of New Orleans, mbethel@uno.edu \\ Lynn F. Brien \\ University of New Orleans \\ Emily J. Danielson \\ University of New Orleans \\ Shirley B. Laska \\ University of New Orleans \\ John P. Troutman \\ LA Coastal Protection and Restoration Authority
}

See next page for additional authors

Follow this and additional works at: https://scholarworks.uno.edu/chart_pubs

\section{Recommended Citation}

Bethel, Matthew B.; Brien, Lynn F.; Danielson, Emily J.; Laska, Shirley B.; Troutman, John P.; Boshart, William M.; Giardino, Marco J.; and Phillips, Maurice A., "Blending Geospatial Technology and Traditional Ecological Knowledge to Enhance Restoration Decision-Support Processes in Coastal Louisiana" (2011). CHART Publications. Paper 23.: $555-571$

https://scholarworks.uno.edu/chart_pubs/23

This Article is brought to you for free and open access by the Center for Hazards Assessment, Response and Technology (CHART) at ScholarWorks@UNO. It has been accepted for inclusion in CHART Publications by an authorized administrator of ScholarWorks@UNO. For more information, please contact scholarworks@uno.edu. 
Authors

Matthew B. Bethel, Lynn F. Brien, Emily J. Danielson, Shirley B. Laska, John P. Troutman, William M. Boshart, Marco J. Giardino, and Maurice A. Phillips 


\title{
Blending Geospatial Technology and Traditional Ecological Knowledge to Enhance Restoration Decision-Support Processes in Coastal Louisiana
}

\author{
Matthew B. Bethel ${ }^{\dagger}$, Lynn F. Brien ${ }^{\dagger}$, Emily J. Danielson \\ University of New Orleans \\ Pontchartrain Institute for Environmental \\ Sciences \\ 2045 Lakeshore Drive, CERM Building 360 \\ New Orleans, LA 70148, U.S.A. \\ mbethel@uno.edu \\ ${ }^{\dagger}$ NASA Stennis Space Center \\ Applied Science and Technology Project \\ Office \\ Stennis Space Center, MS 39529, U.S.A. \\ University of New Orleans \\ Center for Hazards Assessment, \\ Response, and Technology \\ Milneburg Hall, Room 100 \\ New Orleans, LA 70148, U.S.A. \\ \#The Community of Grand Bayou, \\ Louisiana \\ Grand Bayou Road \\ Port Sulphur, LA 70083, U.S.A.
} John P. Troutman ${ }^{\S}$, William M. Boshart ${ }^{\S}$, Marco J. Giardino ${ }^{\dagger \dagger}$, and Maurice A. Phillips

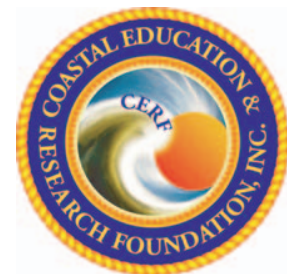

www.cerf-jcr.org

${ }^{s}$ Louisiana Coastal Protection and Restoration Authority

Office of Coastal Protection and Restoration 2045 Lakeshore Drive, CERM Building,

Suite 309

New Orleans, LA 70122, U.S.A.

\begin{abstract}
BETHEL, M.B.; BRIEN, L.F.; DANIELSON, E.J.; LASKA, S.B.; TROUTMAN, J.P.; BOSHART, W.M.; GIARDINO, M.J., and PHILLIPS, M.A., 2011. Blending geospatial technology and traditional ecological knowledge to enhance restoration decision-support processes in coastal Louisiana. Journal of Coastal Research, 27(3), 555-571. West Palm Beach (Florida), ISSN 0749-0208.

More informed coastal restoration decisions have become increasingly important given limited resources available for restoration projects and the increasing magnitude of marsh degradation and loss across the Gulf Coast. This research investigated the feasibility and benefits of integrating geospatial technology with the traditional ecological knowledge (TEK) of an indigenous Louisiana coastal population to assess the impacts of current and historical ecosystem change on community viability. The primary goal was to provide coastal resource managers with a decision-support tool that allows for a more comprehensive method of assessing localized ecological change in the Gulf Coast region, which can also benefit human community sustainability. Using remote sensing (RS) and geographic information systems (GIS) mapping products, integrated with a coastal community's TEK to achieve this goal, the research team determined a method for producing vulnerability/sustainability mapping products for an ecosystem-dependent livelihood base of a coastal population based on information derived from RS imagery prioritized with TEK. This study also demonstrates how such an approach can engage affected community residents who are interested in determining and addressing the causes and mitigating the decline of marsh habitat.

Historical image data sets of the study area were acquired to understand evolution of land change to current conditions and project future vulnerability. Image-processing procedures were developed and applied to produce maps that detail land change in the study area at time intervals from 1968 to 2009. This information was combined in a GIS with acquired TEK and scientific data sets relating to marsh vegetation health and vulnerability characteristics to produce mapping products that provide new information for use in the coastal restoration decision-making process. This information includes: (1) marsh areas that are most vulnerable; and (2) the areas that are most significant to community sustainability.
\end{abstract}

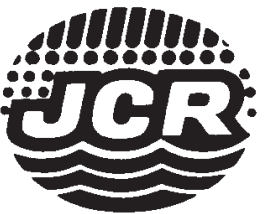

ADDITIONAL INDEX WORDS: Restoration, GIS, remote sensing, traditional ecological knowledge, coastal Louisiana, land loss, marsh health, community vulnerability, Grand Bayou, ecosystem users, coastal management.

\section{INTRODUCTION}

Louisiana's coastal wetlands serve as essential buffer zones between land and water in estuaries and coastal zones; however, they are disappearing rapidly, and those that remain are often in poor health. The most dramatic coastal marsh losses in the United States are in the northern Gulf of Mexico,

DOI: 10.2112/JCOASTRES-D-10-00138.1 received 16 September 2010; accepted in revision 1 November 2010.

Published Pre-print online 1 February 2011.

(c) Coastal Education \& Research Foundation 2011 which has $41 \%$ of the nation's coastal wetlands (Turner, 1997). Louisiana's rate of coastal wetland loss reached a peak of $108.4 \mathrm{~km}^{2} / \mathrm{y}$ in the $1970 \mathrm{~s}$ (Barras et al., 2003). Since the $1980 \mathrm{~s}$, this peak rate of marsh loss has declined (Britsch and Dunbar, 1993), but the trend of land loss continues, with projected loss over the next 50 y estimated to be over $1200 \mathrm{~km}^{2}$ (Barras et al., 2003). The remaining marsh areas serve as a cushion between coastal communities and the open water of the gulf, as well as an integral resource for the economic and social viability of these communities, by supporting the fisheries and coastal/ offshore oil and gas industries, which account for a significant 
portion of the coastal population's employment (Gramling and Hagelman, 2005). These coastal marshes are also critical physical buffers against the full fury of storm events that impact the more densely populated areas in the gulf region, such as the Greater New Orleans Metropolitan Area (Freudenburg et al., 2009). Therefore, coastal community leaders, government officials, and resource managers must be able to accurately assess and predict a given coastal community's sustainability and/or vulnerability as this coastal habitat continues to undergo rapid and dramatic changes associated with natural processes and anthropogenic activities, as well as coastal restoration efforts.

The dependency of coastal communities and more populated areas inland on the marshes was clearly illustrated during the 2005 hurricane season as the Louisiana Gulf Coast bore the brunt of Hurricanes Katrina and Rita. The destructive impact of these storms to coastal communities and populated centers inland was more pronounced after decades of loss of critical marsh habitat. It is hypothesized that a storm surge approaching New Orleans from the south through existing coastal marshes could have been reduced by $3.7 \mathrm{~m}$ if it had crossed $80 \mathrm{~km}$ of marsh before reaching the city (Mitsch and Gosselink, 2007).

\section{Current Coastal Restoration Management}

Since the 1980 s, the state of Louisiana, in partnership with various federal agencies, has been implementing wetland restoration projects to slow the rate of wetland loss. The main types of projects include freshwater diversions (i.e., river reintroductions), hydrologic restoration (marsh management), marsh creation with dredged materials, and barrier island restoration. Under the Coastal Wetlands Planning Protection and Restoration Act (CWPPRA), project ideas are evaluated, and several projects are selected for funding each year. Selected projects go through extensive planning, design, construction, and then operations, maintenance, and monitoring. Constructed projects are periodically evaluated, and the project effectiveness results are fed back into the planning process.

Coastal restoration planning tools have tended to focus on biophysical characteristics of the area of interest. For example, the Wetland Value Assessment (WVA) is a heavily used component of the current coastal restoration decision-making process. It provides an estimate of the number of acres benefited, enhanced, or restored by a proposed project (CWPPRA, 2006). Variables considered to be important in characterizing fish and wildlife habitat are entered into the WVA model, and a Habitat Suitability Index (HSI) is produced. The HSI ranges from 0.1 to 1.0 and is a numerical representation of the overall habitat quality of the particular wetland being evaluated. Other physical data used in the planning and evaluation phases include remotely sensed imagery (Folse et al., 2008; Steyer et al., 2000). Specifically, these image data sets are used to calculate land loss, among other physical properties of marsh condition. The land-loss maps typically produced for the Louisiana Coastal Protection and Restoration Authority (CPRA) use Landsat 30-m-resolution images for assessing loss from the regional scale down to projects a few thousand acres in size. These data sets are suitable for regional assessment of land and water trends; however, they may not be appropriate for small project assessments.

More recently, CPRA's Office of Coastal Protection and Restoration (OCPR) has been developing a Project Prioritization Model (PPM) to support decision making regarding the selection of proposed restoration projects. This model uses mathematical calculations to prioritize proposed projects based on the State Master Plan objectives: (1) reduce economic losses from storm-based flooding; (2) promote a sustainable coastal ecosystem; (3) provide habitats for commercial and recreational activities; and (4) sustain the unique heritage of coastal Louisiana (CPRA, 2009). The PPM is designed to be an adaptable tool that allows for the inclusion of new information as it becomes available. As the data driving the model improves, the model's results will help OCPR decide how to better prioritize future restoration and protection projects (CPRA, 2009). In fact, OCPR encourages ideas from the public as to how this model and other restoration tools can be improved.

\section{The Role of Local Knowledge in Coastal Restoration}

Whereas the WVA has proven useful in assessing potential impact to the habitats of fish and wildlife during the coastal restoration planning process, little effort has been made to understand the social and cultural interpretations of restoration within which the biophysical resources are embedded. This is because scientific models, such as those used for restoration planning and assessment, are typically built to utilize only scientific knowledge, which is derived from systematic observations and experiments that target selective environmental factors. However, use of only scientific knowledge to inform restoration management and planning inhibits the ability of the decision maker to effectively deal with differences in local conservation priorities, or to collectively consider the social, cultural, and political impacts of restoration (Balram, Dragicevic, and Meredith, 2004).

Traditional ecological knowledge (TEK) is defined as a cumulative body of knowledge, practice, and belief that evolves by adaptive processes, is handed down through generations by cultural transmission, and centers on the relationships of humans with one another and with their environment (Berkes, Colding, and Folke, 2000). Examples of TEK include: impacts of historical land loss on affected communities; changes in flora and fauna; natural resource use, and degradation of those resources over time; a history of man-made structures and impacts to the ecosystem and community; and the identification of priority areas of community significance or concern. A substantial body of TEK exists in Louisiana's coastal communities, particularly in the indigenous groups that have historically lived in the marshes and make their living directly from the ecosystem services offered by those marshes. That source of information is virtually unused in the restoration planning process. One reason for the underutilization of TEK is likely because qualitative TEK data are not readily compatible for input into mathematical models, such as the habitat variables of the WVA. Nonetheless, there is evidence that restoration managers and scientists are beginning to recognize the value of incorporating TEK into the current decision- 
support system. For example, the Louisiana Coastal Area Science and Technology Program (LCA S\&T), which informs the use of coastal restoration strategies in Louisiana, recommended support of "research that focuses on local ecological knowledge" noting that, "such knowledge can inform scientific discourse and lead to improved project planning" (LCA S\&T, 2010, p. 4).

\section{Rationale for Data Integration to Support Decision Making}

Studies such as those conducted by Petch, Oauknerova, and Heywood (1995) and Balram, Dragicevic, and Meredith (2004) have shown that there are many benefits to integrating TEK and scientific knowledge in a geographic information systems (GIS) spatial framework; these include: incorporating inputs and policies at various levels of spatial aggregation; promoting spatial and temporal thinking about issues and concerns; and creating opportunities for learning and sharing of responsibilities. However, there are no examples of applied spatial knowledge integration research from scientific and local knowledge sources to inform coastal restoration decision making. In order to demonstrate the way in which this knowledge fusion may be used to enhance the current restoration decision-making process, this study presents a collaborative GIS method for integrating TEK and scientific knowledge with spatial environmental data in an interactive participatory process for establishing restoration priorities, and it demonstrates how this knowledge fusion may be used to enhance current restoration decision-making processes. The integrated data set allows the local and technical knowledge experts to share, explore, manage, analyze, and interpret the multidimensional data in a standard spatial context in order to develop more informed restoration decisions.

The goal of this research was to develop a wetland restoration planning decision-support tool that incorporates scientific data sets and TEK to provide a more comprehensive method of assessing ecological change that can benefit both ecosystem and human community sustainability. The objectives were to: (1) produce historical land-cover change and marsh condition maps of a degraded wetland using remote sensing (RS), GIS, and in-situ marsh biophysical data; (2) record, document, and analyze the TEK of local indigenous residents whose livelihoods are dependent on the surrounding ecosystem; and (3) integrate both sources of information (scientific and traditional) into a GIS. This research represents an unprecedented effort to merge diverse spatial, biophysical, and traditional knowledge regarding marsh condition into a format suitable for informing current coastal restoration decision-support processes at a resolution suitable for localized decision making. It also engages users directly in the process of analyzing the marsh changes and effects in anticipation of restoration efforts.

\section{STUDY AREA}

The focus of this study is the approximately $71 \mathrm{~km}^{2}\left(27.4 \mathrm{mi}^{2}\right)$ area encompassing the ecological livelihood base of the coastal community of Grand Bayou in lower Plaquemines Parish,

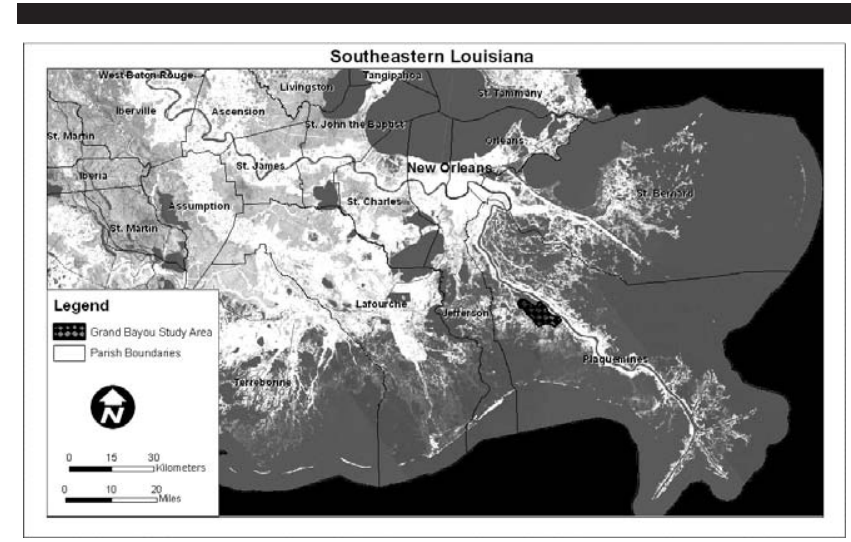

Figure 1. Map of SE Louisiana showing location of the Grand Bayou study area and parish boundaries.

Louisiana (Figure 1). Geographically, Grand Bayou is located within or near several ongoing and planned CWPPRA marsh restoration projects that either have affected or will impact the community and its ecological livelihood base. This area has experienced some of the highest rates of land loss in the Louisiana coastal zone. For the area near Grand Bayou (as calculated for the Pointe a la Hache Digital Orthophoto Quarter Quadrangle by Dunbar, Britsch, and Kemp, 1992), the average rate of marsh land loss was $0.73 \mathrm{~km}^{2} / \mathrm{y}$ between 1932 and 1958, $1.94 \mathrm{~km}^{2} / \mathrm{y}$ between 1958 and $1974,1.84 \mathrm{~km}^{2} / \mathrm{y}$ between 1974 and 1983, and $1.94 \mathrm{~km}^{2} / \mathrm{y}$ between 1983 and 1990.

A project specifically related to this research is BA-04-West Pointe a la Hache Outfall Management. The status of this CWPPRA restoration project is detailed on the Louisiana Coastal Wetlands Conservation and Restoration Task Force Web site (LaCoast, 2008), which contains information and links relating to restoration projects in coastal Louisiana. The West Pointe a la Hache freshwater diversion siphons have been operational since early 1993 and divert sediment and freshwater from the Mississippi River into the surrounding marshes.

The residents of Grand Bayou, who are self-identified as predominantly Native Americans of the Atakapa tribe, with a mix of various other cultures to a lesser degree, trace their ancestry within this region back 200 to 300 y. Community members have historically sustained themselves by utilizing natural resources, particularly the marine resources, available to them in their coastal environment, a tradition that persists in the Atakapa culture today. Like generations before them, the residents are fishers, hunters, and trappers who depend upon the natural resources of the surrounding ecosystem to sustain their way of life. They utilize the local ecosystem in the seasonal harvest of all commercial species including shrimp, oysters, crab, softshell crab, nutria, alligator, and fish. Grand Bayou residents are intricately tied to the surrounding ecosystem and have a long history of adaptation to the challenges associated with persistent change within the ecosystem due to both natural and anthropogenic factors. Because of their dependency upon the environment, the 
residents are actively engaged in issues such as habitat restoration, water quality, and economic development to promote conservation and understanding of this complex and vulnerable ecosystem. Oral history dominates their culture, and as a result, their knowledge of the ecosystem derives both from extensive personal experience and from TEK, the cumulative body of knowledge handed down through generations. This knowledge makes them valuable partners in restoration planning.

Since Hurricane Katrina (which had the most devastating impact to Grand Bayou of any storm in living memory as reported by the residents), efforts at resource utilization have become increasingly difficult due to displacement of many community members and the significant obstacles the community faced in the rebuilding process. The residents of Grand Bayou recognize the importance of rapid and effective marsh restoration so that community members can return and continue to live where they are able to successfully sustain their traditional livelihood of natural resource harvest activities and retain their endemic understanding of this constantly changing area.

\section{DATA AND METHODS}

Using RS, GIS, and other geospatial technologies complemented by a coastal community's TEK, we created a detailed assessment of historical land loss in the study area and evolution of the landscape to its current condition, a method for producing a marsh surface condition map that presents overall marsh health and potential for deterioration, as well as a method for mapping TEK-based information. These data sets were combined in a format that can provide a more comprehensive assessment of ecological change than is currently utilized in restoration decision making that includes effects on local resource utility value and areas of cultural significance.

Studies of the location and historical rates of land loss in coastal Louisiana are often limited to change in spatial extent; however, prioritization of future restoration efforts requires additional information regarding marsh condition. There are many natural and anthropogenic factors that contribute to marsh degradation and loss. The initial TEK data collection indicated that there were two main driving factors related to land loss in the study area: (1) marsh vegetation health, and (2) marsh fragmentation. As a result of this information, the parameters investigated for evaluating potential land loss in this area were related to biophysical characteristics and spatially dependent relationships within the landscape. Marsh biophysical characteristics include the distribution of chlorophyll content, leaf area index, vegetation fraction, and biomass. These biophysical characteristics are indicators of the physiological status of marsh vegetation. Monitoring of these characteristics through remotely sensed imagery can aid in the inference of the overall health of these areas so that more informed restoration management strategies may be implemented. Spatially dependent relationships refer to the patterns of change related to the configuration and connectivity of landcover types within a landscape. Prediction of future change can be aided through better understanding of the spatial relationships of land-cover types for a given area.
It is well documented that most physiological stress in plants will reduce the concentration of photosynthetic pigments, and as a result stressed plants are known to have different spectral reflectance characteristics compared to healthy ones (Nilsson, 1995). Various vegetation indicies can serve as indicators of plant health and chlorophyll pigment loss. For instance, Vigier, Pattey, and Strachan (2004) reported that plant damage was associated with the chlorophyll absorption in reflectance and normalized vegetation indicies, showing a loss of chlorophyll pigment compared to healthy plants. Carter and Spiering (2002) determined specific wavelengths that are most sensitive to chlorophyll concentration in an effort to better understand the relationship between leaf optical properties and chlorophyll content. Therefore, relative chlorophyll content was used as a biophysical parameter to assess marsh health variability for this study.

Leaf area index (LAI) is the ratio of total upper leaf surface of vegetation divided by a given surface area of the land on which the vegetation grows. Because LAI most directly quantifies the plant canopy structure, it is highly related to a variety of canopy processes, such as water interception, evapotranspiration, photosynthesis, respiration, and leaf litterfall. LAI "is a critical variable for understanding the biological and physical processes associated with vegetated land surfaces" (Wang et al., 2004, p. 114). Given that LAI has been shown to be important in understanding many aspects of plant canopy development, growth, and management, it was the second biophysical parameter, along with chlorophyll content, used in this study.

The quantification of landscape pattern allows us to identify interactions among spatial patterns and ecological processes. Because land-cover maps derived from remotely sensed imagery only indicate the location and type of land cover, further processing is needed to quantify and map land-cover fragmentation (Gustafson, 1998; Turner and Gardner, 1991). Practical applications of landscape pattern quantification include: describing how a landscape has changed through time; making future predictions regarding landscape change; and evaluating alternative land management strategies in terms of the landscape patterns that may result. The calculation of landscape pattern metrics is necessary to rigorously describe landscape patterns (Gergel and Turner, 2003). The purpose of a landscape fragmentation analysis is to map the types of fragmentation present in a land-cover type (i.e., marsh). Turner et al. (2003 p. 3) define fragmentation as the "breaking up of a habitat or cover type into smaller, disconnected parcels." Fragmented land cover is typically classified into four main categories: patch, edge, perforated, and core. These fragmentation types are defined by ESRI (2010) as:

- perforated-pixels along the edge of an interior gap in a land cover that are degraded by edge effects;

- edge-pixels along the exterior perimeter of a land cover that are degraded by an edge effect;

- patch-small isolated fragments of a land cover that are completely degraded by edge effects; and

- core-land-cover pixels that are not degraded by edge effects. 
Table 1. Imagery used in land-change analysis.

\begin{tabular}{|c|c|c|c|}
\hline Image Date & Image Type & Image Source & Image Resolution \\
\hline $11 / 25 / 1968$ & BW scanned aerial photography & U.S. Army Corps of Engineers, New Orleans & $1: 30,000$ (600 dpi) \\
\hline 03/26/1979 & CIR scanned aerial photography & NASA/UL Lafayette Regional Application Center & $1: 65,000(1,500 \mathrm{dpi})$ \\
\hline $11 / 05 / 1991$ & Digital orthophoto quarter quadrangles (DOQQ) & $\begin{array}{l}\text { USGS Earth Resources and Observation Science } \\
\text { (EROS) Center }\end{array}$ & $1 \mathrm{~m}$ \\
\hline 01/24/1995 & CIR scanned aerial photography & NASA/UL Lafayette Regional Application Center & $1: 65,000(1,500 \mathrm{dpi})$ \\
\hline 01/24/1998 & DOQQ & EROS & $1 \mathrm{~m}$ \\
\hline $01 / 21 / 2004$ & DOQQ & EROS & $1 \mathrm{~m}$ \\
\hline $10 / 27 / 2005$ & DOQQ & EROS & $1 \mathrm{~m}$ \\
\hline $10 / 30 / 2008$ & DOQQ & EROS & $1 \mathrm{~m}$ \\
\hline $10 / 30 / 2009$ & Satellite imagery & DigitalGlobe Quickbird & $2.39 \mathrm{~m}$ \\
\hline $11 / 12 / 2009$ & Satellite imagery & DigitalGlobe Quickbird & $2.39 \mathrm{~m}$ \\
\hline
\end{tabular}

Fragmentation type is determined by proximity to fragmenting features (such as water in this case). Originally, fragmentation analysis was developed for use on forest land covers but can be applied to any land cover of interest (ESRI, 2010).

\section{Mapping Historical Land Change}

\section{Preclassification Image-Processing Procedures}

Historical land-loss assessment of the study area utilized high-resolution $(<2.39 \mathrm{~m})$, multitemporal aerial and satellite image data sets spanning a period of approximately $40 \mathrm{y}$, from 1968 to 2009 (Table 1). The image data sets selected for the study were all acquired between fall and early spring, thus minimizing confusion in land-water discrimination caused by the presence of floating aquatic vegetation more common during summer months (Barras et al., 2003). Image processing and analysis were accomplished using ERDAS Imagine 9.3 software. The images were georectified and then resampled as necessary to a common spatial resolution of $2.39 \mathrm{~m}$ using the nearest neighbor method. To ensure uniform georegistration, image data sets were projected to the Universal Transverse Mercator (UTM) Zone 15 North American Datum (NAD) 83 coordinate system. Image frames of scanned aerial photos were mosaicked using an image overlay function, and all image data sets were subset to the study area boundaries. Radiometric enhancement in the form of adaptive filtering noise reduction was applied to the 1968, 1979, and 1995 aerial photos prior to classification. This technique preserved the subtle details in the scanned images while removing noise resulting from the digitization process, as manifested by a grainy appearance. Image-quality issues in the 1968, 1979, 1991, and 2004 data sets resulted in difficulties with classification of land and water in some areas. As a result, subsets were used during or prior to classification to isolate these problematic areas for further processing. Subsets were necessary to isolate areas of sun glint, to address image vignetting, and to deal with the effects of brightness differences along mosaic seam lines (typically affecting $<20 \%$ of the total image area). Cloud masking necessary in the October 30, 2009 image was accomplished by creating a thematic layer in which the pixel values unique to the clouds in that image were grouped into a "cloud" class, which was subsequently recoded to zero to exclude cloud areas that corresponded to $6 \%$ of the total image area.

\section{Image Classification}

Preprocessed image data sets were classified using a standardized methodology based on a hybrid approach of level slicing, and supervised and unsupervised classification techniques. These techniques were combined with recoding of resulting thematic images to derive land-water binary maps for each data set, as well as a vegetation-non-vegetation binary map for the October 30, 2009, data set.

In the supervised classification technique, areas of interest (AOIs) were developed for use as training clusters in the creation of unique spectral signatures for each of the classes. For this process, the eight neighborhood mode was used to determine the pixels that would be considered contiguous (i.e., similar in value) to the selected pixel. The spectral Euclidian distance (the spectral distance from the mean of the seed, or selected pixel) was kept as low as possible, with the goal of obtaining representative training clusters with standard deviations of 3 or less and consisting of a minimum of 25 pixels. This convention was maintained whenever possible, although it was at times necessary to accept slightly higher standard deviation values, depending upon image quality. While a minimum number of 10 training signatures was obtained for each class, in some images 50 or more training sites were selected to adequately represent the variations within a class. The probabilities of the spectral signatures were normalized prior to the supervised classification, which was based upon a maximum likelihood classifier. A distance file was created in the process and used, along with the resulting supervised classified image, to create a threshold image based on a confidence level of 0.050 and $\chi^{2}$ of 9.490. The threshold image resulted from iteratively identifying class threshold values with an image raster attribute table using the original image data set as reference. Once these class threshold values were determined, all values were recoded as land, water, or no data for each image and for vegetation, nonvegetation, and no data for the October 30, 2009, image. For this analysis, the single-band aerial image files were represented as red-bluegreen (RGB) in Imagine 9.3, with the same pixel value representing each band in the RGB signature (corresponding to image brightness).

Unsupervised classification employed the iterative selforganizing data analysis technique (ISODATA), a clustering method that uses a minimum spectral distance formula to form 
clusters or classes of similar spectra for an image data set. The clusters were initialized from statistics, and the number of classes specified varied between 25 and 75, with fewer classes necessary in images exhibiting clear delineation between land and water classes. Maximum iterations were set at 100, with the convergence threshold set at 0.950 .

When necessary, an unsupervised classification image was used to classify remaining unclassified or undetermined pixels in a threshold image. This was accomplished by overlaying the threshold image on the original image and linking it geographically to an unsupervised classification image. In this way, trial and error and careful examination of the images were used to make the best estimates of appropriate classification of remaining undetermined pixels, and thus enhance classification accuracy. In addition, supervised classification was performed on areas of mixed cluster issues resulting from unsupervised classification to better identify land and water pixels for those problem areas. Also, clusters that were not easily labeled were separated from the rest of the image, and then the classification algorithm was applied again to obtain additional clusters (Jensen et al., 1987). Each final classified image was recoded for three values: unclassified, land, and water.

Postclassification modifications designed to reduce classification error related to noise inherent in the data included a neighborhood analysis, a GIS Analysis Clump procedure, applied to the recoded classified images using four connected neighbors to identify contiguous areas of class values. A GIS Analysis Eliminate procedure was then applied to eliminate "clumps", i.e., small island classes (or noise) within larger classes, using four contiguous pixels or less as the threshold for defining a clump to be eliminated.

\section{Change Analysis}

A multidate postclassification comparison was performed as a means of detecting changes in land-water distribution within the study area during the period between 1968 and 2009. Pairs of land-water classified images were compared pixel by pixel, and each comparison resulted in a change detection matrix of land-water transformations (or lack of transformation) and the production of a categorical map depicting and quantifying land, water, land loss, and land gain.

Critical visual examination of sequential change maps revealed areas where land-cover transformations appeared impermanent, reversible, and possibly cyclical. Pixel by pixel comparisons of successive land-cover change maps allowed the delineation of these areas, which were then characterized as transitional zones. Transitional zones were separated from areas that consistently exhibited land loss or gain throughout all time periods analyzed. These transitory land changes were separated from the actual land-loss class so that only land that was permanently lost from a particular date to present was categorized as "actual land loss." The concept of combining several years of observations as a means of discriminating permanent land loss or gain from transitory loss or gain resulting from episodic events such as hurricanes was suggested in methods used by Barras, Bernier, and Morton (2008), and is the basis of this analysis.
Following the identification of the transitional areas, field investigation was conducted to determine possible causes of these features. The 2005-08 transitional change map was loaded into a handheld computer equipped with global positioning system (GPS) to navigate to several transitional zone locations. These areas tended to be shallow submerged bare land or marsh vegetation, likely emergent during low tidal conditions, and thus highly influenced by meteorological (i.e., wind setup/setdown effects) and tidal conditions at the time of image acquisition.

\section{Accuracy Assessment of Land-Cover Classifications}

Quantitative accuracy assessment of land-cover classification reflects how well the land-cover classes were identified from the source imagery. Consistent with guidelines suggested by Congalton and Green (2009), each land-cover classification map created for this study was assessed for accuracy based on the selection of 150 stratified random points, with a minimum 50 points representing each map class. The error matrix generated for each classified image provides the basis for the overall accuracy statistics and the Kappa coefficient of each classification. An overall accuracy level of $85 \%$ was considered the minimum value for acceptable results (Anderson et al., 1976). All final land-water classifications exceeded this accuracy level threshold.

\section{Mapping Community Restoration Priorities and Observed Change Using TEK}

Acquisition and analysis of TEK for this study were based on collaborative field work methods in which TEK is used in scientific studies to locate study sites, obtain specimens and data, and interpret field observations and results. These methods have been shown to provide an excellent means of interacting with a community for an extended period (Huntington, 2000). In contrast to other social science methodologies that utilize a preplanned survey of questions, collaborative field work generally records subjects' observations as they are made in the field, a technique which allows for a more descriptive and complete account of individual and group memory of environmental change (Manning, 2005). Additionally, information gained over long-term relationships and multiple site visits is more detailed than that collected during a single interview. As a result, TEK collection for this study was a dynamic process responsive to changing environmental conditions and accomplished via site visits, frequent phone calls, and social interactions. For the purposes of this study, the collaborative field work effort included introducing the community to the geospatial technology used for the project so that the ecosystem users (participating Grand Bayou residents) and the physical scientists associated with this project became familiar with the reciprocal knowledge of each group. The ecosystem users learned about the mapping techniques and scientific field data collection from the scientists and were guided by them in understanding it, and the physical scientists learned of the TEK data and were guided by the ecosystem users in understanding it. Also, the two groups learned one another's vocabulary in describing study area features for the sake of successful dialogues. 
Maps of the study area were used for visual aids to knowledge transfer; specifically, map biographies of the local residents were determined to be useful (Calamia, 1999; Ferguson and Messier, 1997) within the ongoing collaboration, especially when choosing sites. For these map biographies, printed maps of the Grand Bayou study area were produced from acquired satellite and aerial imagery and used to establish a relative time line of observed ecosystem changes and to document those changes by plotting them directly onto the map. The printed map was also used to record traditional community names for features and to identify "sensitive areas" or areas of particular concern to the local peoples, such as cultural sites (i.e., burial grounds) and marine or mammal "ecozones" (i.e., fish or animal breeding/spawning areas of particular importance to the community, traditional trapping/fishing areas, etc.).

\section{TEK Sample}

Five Grand Bayou resident fishers/trappers participated in the study. Following community-assisted identification of a primary informant with expertise and in-depth understanding of the study area, the remaining informants were chosen using a snowball sampling method wherein the primary informant recommended additional informants with comparable projectrelated knowledge (Patton, 1990). All informants identified themselves as Native American, specifically of the Atakapa tribe, and had been raised in the village.

\section{TEK Data Collection, Transcription, and Coding}

Proper entrée procedure was followed (West et al., 2008), which included a series of social visits with many community members and composition of a "declaration of principles," which outlined expectations and commitments for all involved for the duration of the project. Verbal permission was obtained to record conversations using a digital voice recorder prior to beginning each session of field sampling, TEK collection, or TEK verification activities. The recorder remained on for the full duration of each data-collection session. A social scientist present at each session transcribed all audible conversation as soon as possible following collection, changing the names of informants to protect their anonymity, and inserting field notes regarding setting, activities, and people present. Transcripts of a total of $53 \mathrm{~h}$ of recorded TEK data were uploaded to Atlas.ti qualitative data analysis software (http://www.atlasti.com/) and coded using inductive coding (Crabtree and Miller, 1992).

Coding of the data was accomplished through a line-by-line review of the transcripts, resulting in the creation of themes designed to identify underlying concepts within the data. Direct quotations from the transcribed data sets were used to support and illustrate the themes. Relationships between codes were identified given the interconnected nature of cultural and environmental factors. Segments of the transcripts were also linked to in-field photos and maps used in the field to provide the coder with a visual reference relating to the context of the linked conversation. Linkages were subsequently used to identify emerging themes within the TEK relative to the local landscape.

\section{Theme and Codebook Development}

Procedures for developing TEK themes and a formal codebook were based on methods outlined by Kurasaki (2000) and included annotating the transcribed text, sorting the annotation list, labeling thematic categories, and refining the theme list. Annotations consisted of brief notes summarizing the main points expressed throughout the transcribed data set. These annotations were used to identify themes that emerged during this process. Using the Atlas.ti software, excerpts of the raw data were linked to these annotation themes to serve as examples of each annotation in the list.

The codebook was developed by listing all of the codes that emerged from the TEK data and noting brief definitions for each. For the purposes of synthesis with mapping practices, the codes were organized into two groups, geographic codes and social science codes. Geographic codes represented mappable locations (specific geographic areas that can be depicted on a map). Social science codes represent informants' observations regarding marsh conditions (i.e., descriptor variables used to characterize attributes associated with mappable locations), specific events that represent factors contributing to marsh decline and that inform the project as background information, and sensitive areas of the ecosystem relative to work, quality of life, and cultural significance. Throughout codebook development, preliminary results were verified by contributing informants in a format that could be easily assessed for accuracy (i.e., maps and verbal summaries, as opposed to the complete coded transcriptions).

\section{Intercoder Reliability Assessment}

Intercoder reliability assessment was undertaken to ensure minimization of coder bias or random error arising from judgments made about categories and themes emerging from the complex qualitative data sets. Intercoder reliability is a quantitative measure of agreement between multiple coders with regard to the ways in which codes are applied to TEK data (Kurasaki, 2000). The procedures used to determine intercoder reliability for this study are based upon recommendations by Hruschka et al. (2004) and Kurasaki (2000). First, steps were taken to familiarize a second social scientist with the project and associated TEK data. Due to the volume of TEK data and time constraints, the second coder was presented with approximately 10 pages of randomly selected textual "idea units" from the TEK data, instead of the entire TEK data set as suggested by Hruschka et al. (2004). After the second coder applied the same systematic coding process using the codebook as a guide, results from the two independent coders were compared, and the agreement between the coders was calculated.

Calculation of intercoder reliability agreement involved the random selection of 20 lines per coded page. The results of the two coders were checked for agreement using the randomly selected line plus five lines above and below the selected line. The inclusion of the additional lines is designed to accommodate the expected variation between coders with respect to their identification of TEK relating to specific codes (Kurasaki, 2000). Because of the conversational nature of the interviews, it 
was common to find peripheral text surrounding more substantive, codable text. An agreement matrix was developed, and the agreement between the coders was calculated as the ratio of agreements to total random excerpts for each codebook theme. An overall agreement across all the codes was calculated by averaging the agreements obtained for each code (Kurasaki, 2000). The comparison results were then used to revise the codebook as necessary until an acceptable overall agreement value was achieved.

\section{Applying Numeric Codes}

Once acceptable intercoder reliability was established and the codebook finalized, Atlas.ti was used to investigate the passages related to the "Marsh Condition" codes in the transcribed documents in reference to the "Mappable Locations." Based on the TEK information associated with each of these Marsh Condition codes, an attribute value of $+1,-1$, or 0 was assigned to each mappable location.

With regard to community assessment of restoration priority, a value of +1 is indicative of an area that is important or urgent to restore, while a value of -1 indicates an area considered relatively unimportant or not urgent to restore. A value of 0 indicates that the location or feature was mentioned in the TEK, but a determination of its importance is inconclusive. It is important to note that a -1 value does not indicate that an area should not be restored, but rather that, in comparison to other areas, it is a lower priority for the community.

With regard to assessment of observed change, a value of +1 is indicative of a location or feature characterized by a positive or stable condition over time with regard to natural resources used by the community at that location. In contrast, a value of -1 is associated with a location or feature that has undergone negative change or degradation in natural resources over time. A value of 0 indicates that the TEK information is inconclusive for a particular mappable location/feature.

Calculation of the Restoration Priority Index (RP) and the Index of Observed Change (OC) is based on the following:

$$
i=(a-b) x
$$

where $i=$ index, $a=$ number of codes with value of $+1, b=$ number of codes with value of -1 , and $x=$ total number of codes.

The RP and OC indices were used to produce TEK-based maps showing the locations of features identified in the TEK, as well as a community prioritization for the restoration of those features (Figure 2). The maps were presented to the TEK informants for verification and validation of the results, and any discrepancies were corrected prior to final map production.

\section{Mapping Marsh Areas Vulnerable to Loss Using Scientific Data Sets}

\section{Field Data Collection}

Sampling site selection for the scientific marsh biophysical condition data sets was based on accessibility, extent of land loss observed, importance to the community, and availability of

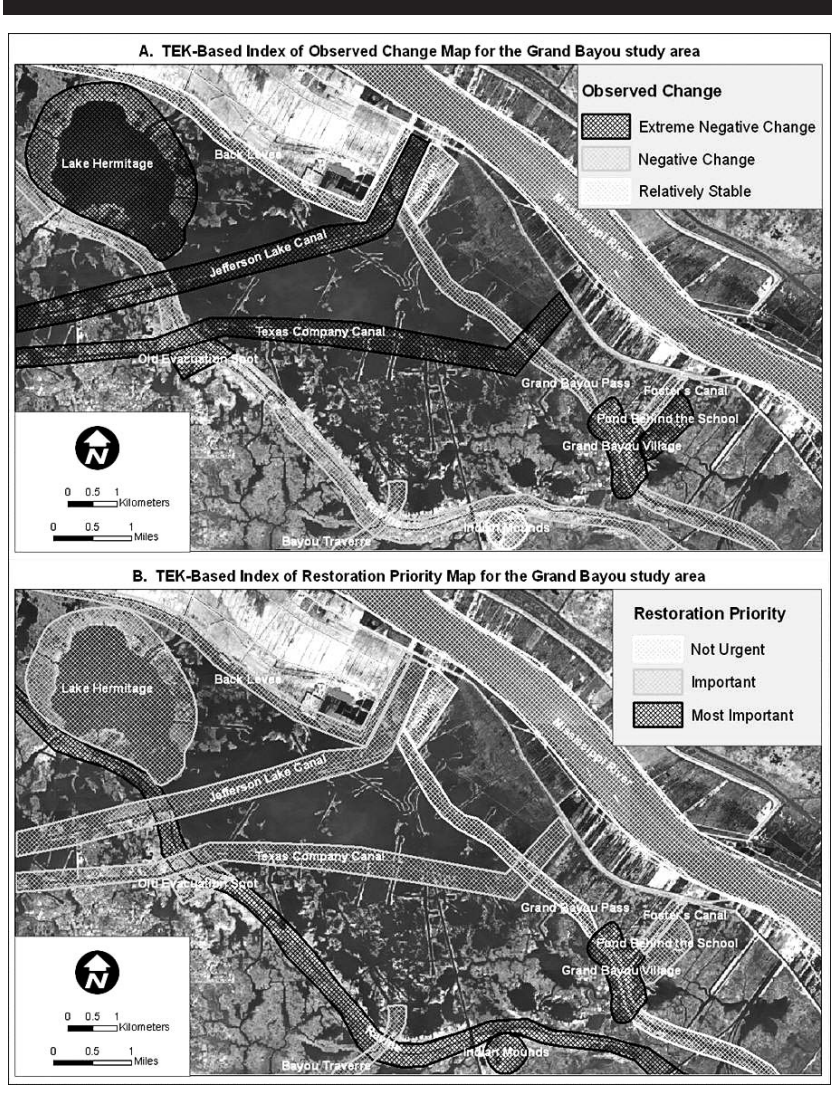

Figure 2. TEK-based indices maps depicting locations of features identified with TEK collected for this study: (A) TEK-based index of observed change map reflecting relative condition over time of these locations as it relates to the natural resources used by the community; and (B) TEK-based index of restoration priority map showing community prioritization for the restoration of these features.

historical data (both TEK and scientific data). Sites were also evaluated with regard to their representation of various marsh conditions observed within the study area. Areas of broken and degraded marsh were chosen to contrast with other selected areas of relatively contiguous, "firm" marsh. Several trial field data-collection campaigns were conducted to test and refine field data-collection methods and to determine logistics relative to site accessibility, data and hardware needs, time required at each site, and personnel requirements for subsequent field sampling. Based on these trials and the field data-collection resources available for this effort, it was possible to sample 20 sites within the $71 \mathrm{~km}^{2}$ area during an approximate $6 \mathrm{~h}$ window of opportunity when the sun angle was most appropriate for spectral reflectance measurements. Subsequent to data collection, two sampling sites were determined to be statistical outliers when examining the data distribution and semiovariogram/covariance cloud for the field data collected. These two sites were considered to be in error and removed from the data set for further analyses. The remaining field data were normally distributed and included observations at 18 sites for chlorophyll content and LAI. Instruments used in the scientific field data sampling included: Ocean Optics USB4000 Field Spectroradiometer to measure in-situ spectral reflectance; LI- 
COR LAI-2000 Plant Canopy Analyzer to measure relative marsh biomass; and the FieldScout CM1000 Chlorophyll Meter to measure relative chlorophyll content.

The Ocean Optics USB4000 spectroradiometer system consisted of two connected spectroradiometers. One measures incoming sunlight and the other measures upwelling light from a target $(\sim 350-1045 \mathrm{~nm}$, at $\sim 0.2 \mathrm{~nm}$ resolution). A white reference panel (made to reflect $99 \%$ of incoming radiation) was also used to calibrate reflectance measurements. The spectrometers were operated using a ruggedized laptop and dataacquisition software. The simultaneous collection of upwelling and incoming radiation compensates for changes in lighting conditions between calibration and data collection.

Top of canopy (TOC) reflectance measurements with the Ocean Optics USB4000 system were made by mounting the fiber optic of the spectroradiometer on a pole to allow for the collection of spectral reflectance data at an offset from the operator. This minimizes any shadows or interference in data collection by the user and allows data to be collected from the top of the vegetation canopy. This configuration results in an approximate 27 inch instantaneous field of view (IFOV). Three reflectance measurements were made within a target datacollection area (DCA) of $10 \mathrm{~m}^{2}$ and then averaged for each sampling site. One calibration measurement with the white reference panel was also made at each sampling location.

The LAI-2000 Plant Canopy Analyzer measures the probability of seeing the sky looking up through a vegetative canopy in different directions. Using these measurements, the LAI2000 calculates foliage amount (LAI) and foliage orientation (mean foliage tilt angle) by measuring the rate at which radiation is attenuated as it passes through the canopy. The LAI calculations require measurements above canopy periodically to calculate total transmittance at the time that belowcanopy measurements are made. In this manner, the LAI readings are calibrated for atmospheric conditions. If sky conditions are stable, one above-canopy measurement will suffice for several subsequent below-canopy measurements (LAI-2000, 1992). For this study, one above-canopy measurement was made for every four below-canopy measurements. At each sampling site, three separate above/below-canopy sampling sequences were made and averaged within a $10 \times 10 \mathrm{~m}$ area.

The FieldScout CM1000 Chlorophyll Meter senses light at wavelengths of $700 \mathrm{~nm}$ and $840 \mathrm{~nm}$ to estimate chlorophyll content in leaves (Spectrum Technologies, 2008). Chlorophyll $a$ absorbs $700 \mathrm{~nm}$ light, and, as a result, the reflection of that wavelength from a leaf is reduced compared to the reflected $840 \mathrm{~nm}$ light. Light having a wavelength of $840 \mathrm{~nm}$ is unaffected by leaf chlorophyll content and serves as an indication of the amount of light that is reflected due to leaf physical characteristics. As each measurement is taken, the result is displayed as an index with a range of 0 to 999 . This index is based on the ratio of $700 \mathrm{~nm}$ light to $840 \mathrm{~nm}$ light reflected from the target, multiplied by a constant. A standard method was used to obtain measurements with the CM1000 that included taking readings with the sun always at the user's back and at an angle of approximately $45^{\circ}$ between the user and the target. Five CM1000 measurements were taken within a 10 $\times 10 \mathrm{~m}$ area and averaged at each sampling location.

\section{Image Calibration}

The field data collected with the Ocean Optics USB4000 at the predefined sampling sites throughout the study area occurred on November 2, 2009, and were the basis for atmospheric correction of the October 30, 2009, image data set acquired for this project. The DCA of the field data collected at each sampling site approximates an area that includes four contiguous pixel values extracted and averaged from the image data set. Image calibration was performed using an empirical line calibration (ELC) between the reflectance values for the field spectroradiometer at each sampling site and the values for the same sites retrieved from the imagery. The ELC method of calibration matches the spectral reflectance of remotely sensed images to in-situ spectral reflectance measurements obtained at approximately the same time as the remote-sensing overflight (Jensen, 2004). The in-situ and remote-sensingderived spectra were regressed, and the values were then applied to the remote-sensor data on a band-by-band basis, removing atmospheric attenuation (Jensen, 2004). Regression analysis was performed between the averaged Ocean Optics spectroradiometer reflectance values and the averaged raw digital number $(\mathrm{DN})$ values at each $10 \times 10 \mathrm{~m}$ sampling site for each Quickbird band. The results are as follows (values are $\mathrm{R}^{2}$ and standard error, respectively): Band $1-0.99 / 0.74$; Band $2-$ 0.99/0.91; Band 3-0.99/1.5; and Band 4-0.98/5.29.

\section{Estimated CM1000 and LAI Maps}

Clouds were masked in the October 30, 2009, image as described in the Preclassification Image-Processing Procedures. This "cloud mask" file was intersected with the original extent of the calibrated October 30,2009, image to mask out the areas obscured by clouds for later analyses. Subsequently, a supervised classification and threshold process was performed on the cloud-masked image using the methods described previously. The resulting classes were recoded as either vegetation or nonvegetation. The nonvegetation areas were then masked out using the same procedure as was used to mask out clouds. The final calibrated, masked October 30, 2009, image data set was used in developing the estimated LAI and chlorophyll maps.

Several vegetation indices and band combinations were derived based upon the averaged reflectance values extracted from the November 2, 2009 reflectance field data that matched the calibrated image data set for each band at each sampling site. The literature identifies several vegetation indices found to be promising in mapping wetland vegetative vigor as it relates to chlorophyll content and LAI (Gitelson, 2004; McCarthy et al., 1993; Ozesmi and Bauer, 2002; Qi et al., 1994; Zhang et al., 1997). Therefore, the vegetation indices tested in this study included: the Normalized Difference Vegetation Index; Ratio Vegetation Index; Green Normalized Difference Vegetation Index; Atmospheric Resistant Vegetation Index; Modified Soil Adjusted Vegetation Index; Modified Triangular Vegetation Index; and Wide Dynamic Range Vegetation Index. These vegetation indices were statistically assessed for correlation with the ground information acquired.

Each vegetation index was produced from the averaged field reflectance data that corresponded to each band in the October 
30, 2009, image. Also, each vegetation index was computed for the calibrated, masked October 30, 2009, image data set. This was done for the reflectance values at each sampling location.

Each field data set (chlorophyll meter and LAI) was divided into a test and validation data set, so that the resulting estimated maps produced with the test data sets could be assessed for accuracy. Ten sampling sites were randomly chosen as the test data set, and the remaining eight sites served as the validation data set for each parameter estimated.

Stepwise multiple regression and multiple correlation analyses were performed on each test data set using SAS software (SAS Institute, 2002) to determine the best band combination/vegetation index to use for each dependent variable tested (chlorophyll meter and LAI values), and to test for the presence of multicollinearity among the variables in the regression model (Cody and Smith, 1991). A statistical regression analysis was performed to ensure that a relationship existed with the imagery, and given that relationship, the image-processing technique determined to have the strongest relationship with the field data collected was used to create the estimated chlorophyll meter and LAI maps. The parameter estimates for the regression equations chosen as the best estimate for each dependent variable were applied to the corresponding October 30, 2009, index image.

The resulting vegetation index ranges for the estimated chlorophyll content and LAI images were used to recode the image into relative categories of vegetative health and produce a classed "relative" marsh health map. The class threshold values were determined based on statistical analyses of the vegetation index values across the study area and consultation with OCPR project collaborators. As a result, a three-class Natural Breaks (Jenks) classification of the continuous data values for each estimated map was used for display and recoded as high, medium, and low relative marsh health classes.

\section{Fragmentation Map Production}

The final land-water image for each historical image date was used to produce fragmentation maps. This was accomplished with the Landscape Fragmentation tool in ArcGIS 9.3, which testing has shown to be equivalent to procedures used by Vogt et al. (2007). For the purposes of this study, landscape fragmentation classes based upon research by Vogt et al. (2007) were used for mapping spatial patterns and further refined by consultation with the collaborating OCPR scientists with the aim of creating a mapping product that could be easily integrated into their existing decision-support system. As a result, the land class in each image was further classified into six categories: perforated, edge, patch, small core ( $<250$ acres $)$, medium core (between 250 and 500 acres), and large core ( $>500$ acres). An edge width parameter was specified as $15 \mathrm{~m}$. The literature indicates that the edge width varies according to the issue of interest (Riiters et al., 2000; Vogt et al., 2009). This edge effect distance was chosen after testing several different values for this parameter, and based on visual examinations of the resulting fragmentation maps and input data sets, it was decided that $15 \mathrm{~m}$ represented the optimum distance for edgeeffect influence for this data set.

The fragmentation maps were then combined in ArcGIS 9.3 with the corresponding historical land-change image. For instance, the 2009 fragmentation map was combined with the 2005-09 land-change image. The resulting combined images show areas where the land-loss class intersects the fragmentation classes. These areas of intersection were then reclassed in the combined images as: patch to land loss; edge to land loss; perforated to land loss; core 1 to land loss; core 2 to land loss; and core 3 to land loss. Area was calculated for each of these classes in the combined image, and then the proportion of the total area that went to land loss was calculated for each fragmentation class for each historical image date.

The proportions of each fragmentation class that went to land loss for each date were statistically analyzed in SAS with a General Linear Model (GLM) to determine any significant difference that existing among the land-loss proportions of the fragmentation classes. A Duncan's multiple comparison test was performed to further investigate any differences existing between the fragmentation classes (Cody and Smith, 1991).

\section{RESULTS}

\section{Historical Change}

Six final change maps were produced depicting the transitional area class, as well as actual land-loss and actual land-gain classes. For each of these maps, the total area in hectares (and acres) was calculated for each class. Net land loss and the average land loss per year were then calculated for each time period represented. The results for each composite map are shown in Table 2, and Figure 3 shows a composite map that represents actual land loss for each of six change maps produced.

The land-to-water ratio was calculated for each land-water classification date. The results show that the proportion of land to water in the study area consistently decreased from 1968 to 2009. The proportion of total land to total water in the study area can be summarized as follows: 2.62 in 1968; 1.57 in 1979; 0.79 in $1991 ; 0.71$ in $1998 ; 0.65$ in $2004 ; 0.61$ in 2005 ; and 0.48 in 2009. The proportion of total land to water area for each historical image date is shown in Figure 4.

\section{TEK-Based Mapping Results}

The intercoder reliability assessment for the coded transcripts resulted in a $98 \%$ overall agreement between the two coders in assigning codes to 20 randomly selected text segments from the transcribed TEK data sets. This finding strengthens the validity of the coding results, as well as the conclusions based on the coded text data, and demonstrates that subjectivity in the coding process was minimized.

Identification of specific areas of concern was difficult for some informants because they emphasized that the entire study area was vital and contributed to their safety and lifestyle sustainability. Specifically, they rely on the health of the entire marsh for protection during hurricanes and tropical storms and as their primary means of sustaining themselves through shrimping, oystering, and fishing. It was emphasized that every area is important to restore because the whole ecosystem works together. Therefore, the reader should not assume that a low-priority area should not be addressed. The entire area represented on the RP map and beyond has been 
Table 2. Land loss by time periods and hurricanes that passed within 65 miles of study area.

\begin{tabular}{|c|c|c|c|c|c|}
\hline Time Period & $\begin{array}{l}\text { Actual Land Loss in } \\
\text { Hectares (acres) }\end{array}$ & $\begin{array}{l}\text { Actual Land Gain in } \\
\text { Hectares (acres) }\end{array}$ & Hurricane Event & $\begin{array}{l}\text { Net Land Loss in } \\
\text { Hectares (acres) }\end{array}$ & $\begin{array}{l}\text { Average Land Loss Per } \\
\text { Year in Hectares (acres) }\end{array}$ \\
\hline $\begin{array}{l}11 / 25 / 1968-03 / 26 / 1979 \\
\quad(\sim 10 \mathrm{y}, 4 \mathrm{mo})\end{array}$ & 711 (1758) & $148(367)$ & Camille & $563(1391)$ & $69(170)$ \\
\hline $\begin{array}{l}03 / 26 / 1979-11 / 05 / 1991 \\
\quad(\sim 12 \mathrm{y}, 7 \mathrm{mo}) \\
11 / 05 / 1991-01 / 24 / 1998\end{array}$ & $1042(2574)$ & $148(367)$ & Bob, Florence & 894 (2208) & $83(205)$ \\
\hline $\begin{array}{l}(\sim 6 \mathrm{y}, 3 \mathrm{mo}) \\
01 / 24 / 1998-01 / 21 / 2004\end{array}$ & 392 (969) & $243(600)$ & Danny & 149 (369) & $63(155)$ \\
\hline $\begin{array}{l}(\sim 6 \mathrm{y}) \\
01 / 24 / 2004-10 / 27 / 2005\end{array}$ & $288(711)$ & $167(413)$ & Georges & $121(298)$ & 48 (119) \\
\hline $\begin{array}{c}(\sim 1 \mathrm{y}, 9 \mathrm{mo}) \\
10 / 27 / 2005-11 / 12 / 2009\end{array}$ & $343(848)$ & $199(491)$ & Ivan, Cindy, Katrina & $144(357)$ & $196(484)$ \\
\hline$(\sim 4 \mathrm{y})$ & $397(980)$ & $62(154)$ & Gustav & $335(826)$ & $99(245)$ \\
\hline
\end{tabular}

identified by the community as in need of restoration. However, within that guideline, researchers ranked locations (for both the Restoration Priority and Observed Change indices) given the context of informants' responses using the numeric coding process described previously and assuming limited resources available for restoration. It was then concluded that the areas the community could not live without-their village and their sacred burial site, would represent the upper limit. The resulting TEK-based maps were presented to the informants to ensure that the TEK information was represented properly. In this manner, the maps were validated as accurate representations of the TEK.

The construction of the canals (primarily for oil extraction) in the study area from the 1950 s to the 1970 s emerged as the most frequently mentioned cause of the land loss observed when analyzing the TEK. The informants accept that some land loss is due to natural processes, such as wave and tidal action, and that it also results from episodic storm events. However, they have observed that the average rate of land loss due to these natural erosion processes was greatly accelerated by the construction of the canals.

Throughout the discussions with the Grand Bayou residents, they frequently offered local restoration and mediation pro-

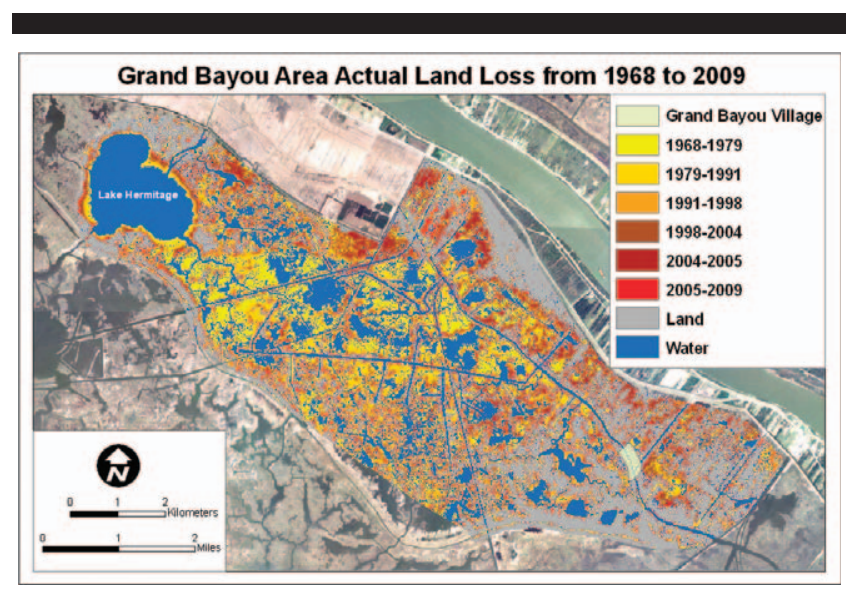

Figure 3. Actual land loss from November 15, 1968, to November 12 , 2009 , by time periods calculated for this study using historical aerial and satellite imagery. posals that they believe could reverse the land-loss trends in the area. The residents' accounts show that they do believe that the rate of land loss can be slowed, and they have hope of reversing some of the damage done to the local ecosystem upon which they depend; however, they expressed little faith in the current restoration policies and programs of authorities. Their skepticism is based on their experience that scientists and restoration managers currently fail to consider their suggestions and knowledge when planning and implementing local restoration projects.

\section{Estimated Chlorophyll and LAI Map Production Results}

The results of the statistical analysis indicate that the best model for estimating chlorophyll meter values is a GVI (green vegetation index) with the following parameter estimates: intercept $=127.94741$, and slope $=217.98764$. The $\mathrm{R}^{2}$ for this model is 0.47 and is significant, with a $P$-value of 0.0278 . A noise-reduction filter was applied to the resulting estimated

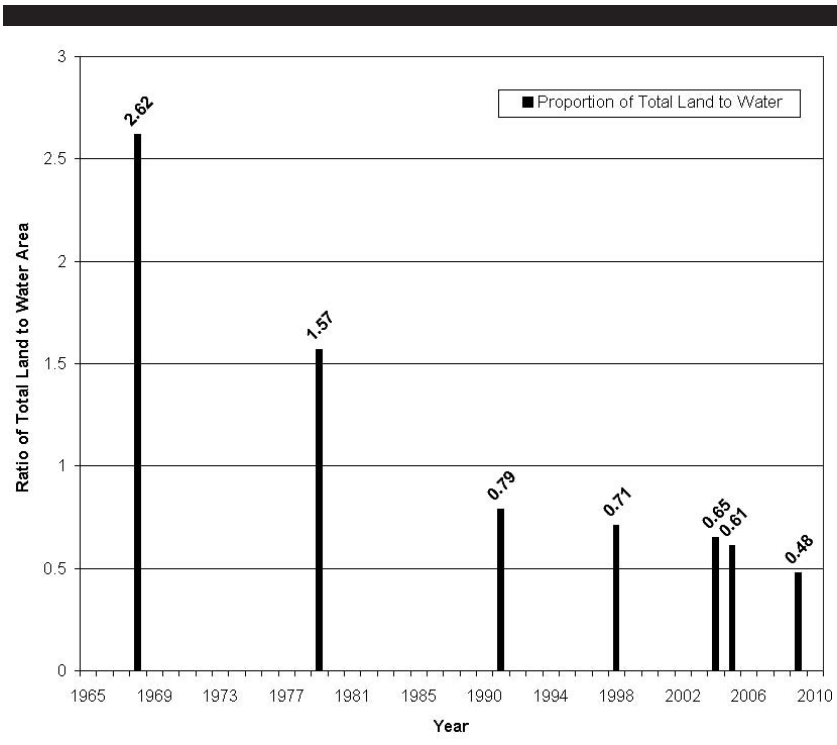

Figure 4. Graph of proportion of total land to water area within the Grand Bayou study area for each date of historical image data used. 
chlorophyll meter map. The standard error resulting from the regression of predicted versus actual values was 31.64 .

The best model for producing the estimated LAI map is an MTV12 (modified triangular vegetation index) with the following parameter estimates: intercept $=-1.84060$, and slope $=6.19449$. The $R^{2}$ value for this model is 0.59 and is significant, with a $P$-value of 0.0098 . A noise-reduction filter was also applied to the resulting estimated LAI map. The standard error resulting from the regression of predicted versus actual values was 0.68 .

The parameter estimates for each dependent variable were applied to the corresponding October 30, 2009, vegetation index image. The resulting index ranges for the estimated chlorophyll content and LAI images were used to recode the image into relative categories and produce classed "relative" marsh condition maps. The chlorophyll content and LAI class threshold values were determined based on statistical analyses of the vegetation index values across the study area and subsequent consultation with OCPR project collaborators. As stated previously, the continuous data values for each estimated map was used for display and recoded as high, medium, and low relative marsh health classes.

Due to delay of field data collection because of persistent adverse weather conditions coinciding with image-acquisition availability, it should be noted that the chlorophyll meter and LAI measurements were collected at a time when the marsh vegetation was nearing fall senescence. The "browning" of the marsh vegetation just prior to fall senescence was visible in certain areas, depending on the dominant marsh vegetation varieties for a particular location. It is expected that this variability in natural senescence occurring at the time of field sampling contributed to the variability in vegetative stress measured and mapped.

\section{Fragmentation Map Production Results}

The results of the GLM statistical analysis showed that the model was significant, with an F-value of 13.38 ( df $=3$ ), and $\operatorname{Pr}>\mathrm{F}=<0.0001$. The Duncan's multiple range test (alpha $=$ 0.05) showed that the Patch class was significantly more likely to be lost than any other class. While the Edge class was significantly more likely to be lost than the Core class, it was not significantly different from the Perforated class. The Core class was significantly less likely to be lost than were either Patch or Edge areas but was not significantly different from Perforated areas.

As a result of this analysis, the 2009 fragmentation map was recoded as follows: Patch class $=$ high risk of loss; Edge class $=$ medium risk of loss; and Perforated and Core areas were combined to make up low-risk areas of loss. This recoded three-class fragmentation map was then combined with the estimated chlorophyll content and estimated LAI maps with the same classification scheme to produce the final marsh vulnerability map.

\section{Merging the Scientific and TEK Data Sets to Enhance Coastal Restoration Decision Making}

To combine the derived data sets to map relative risk of loss, the values of the derived data sets representing estimated chlorophyll content, estimated LAI, and fragmentation class were all reclassified to a common measurement scale (high, medium, and low risk of loss). Values representing water were restricted from this analysis so that only marsh areas would be included. A weighted overlay was performed in ArcGIS 9.3, where the input data sets were assigned percentages of influence. The higher the percentage, the more influence a particular data set has in the resulting map. Because the initial TEK data collection indicated that the two main driving factors related to land loss in the study area were marsh vegetation health and marsh fragmentation, with no clear indication that one factor was more dominant than the other, the estimated chlorophyll content and LAI input data sets were each weighted at $25 \%$, and the fragmentation map was weighted at the remaining $50 \%$. The input data sets were weighted in this way so that the total weighting would be divided equally between the biophysical (chlorophyll content and LAI) and spatial relationship (fragmentation class) measurements.

Pattern analysis was performed on the vulnerability map produced from the weighted overlay of the estimated chlorophyll and LAI maps with the 2009 fragmentation map to determine significantly clustered areas of high land-loss risk. This analysis was performed to determine the probability that spatial clustering was not due to random chance. Cluster and outlier analysis was deemed necessary in order to provide restoration managers with information regarding high-risk areas that result, not from random environmental conditions or from error, but that are instead the result of driving environmental variables. This data can be overlaid on other data sets to determine if a relationship exists between the identified "hot spots" of high risk and areas of land loss. As such, this information can be used to aid restoration decision making and to further investigate causes of marsh degradation experienced in this study area.

The cluster and outlier analysis involved identifying clusters of features with similar values (in this case the three class values, low, medium, and high risk), as well as spatial outliers, using ArcGIS 9.3 to calculate a local Moran's I value, a Z score, a $p$-value, and a code representing the cluster type for each feature. The output data set was queried for all areas corresponding to the high risk of loss class, and subsequently only high-risk areas that were significantly clustered were selected. The resulting selections were output as a separate feature layer and overlaid on the marsh vulnerability map (Figure 5).

\section{Overlay of Marsh Vulnerability Map with TEK-Derived Maps}

GIS overlay, selection by attribute and location, and intersection operations were applied to the derived spatial data sets (clustered high-risk areas for loss, the TEK-based areas of observed change, and the TEK-based areas of restoration priority data layers) to generate detailed maps highlighting areas of high risk that also represent high restoration priorities for the community. This analysis was driven by three criteria: knowledge about risk of land loss derived from scientific data and analyses; knowledge of community-based priorities for continued sustainability; and 


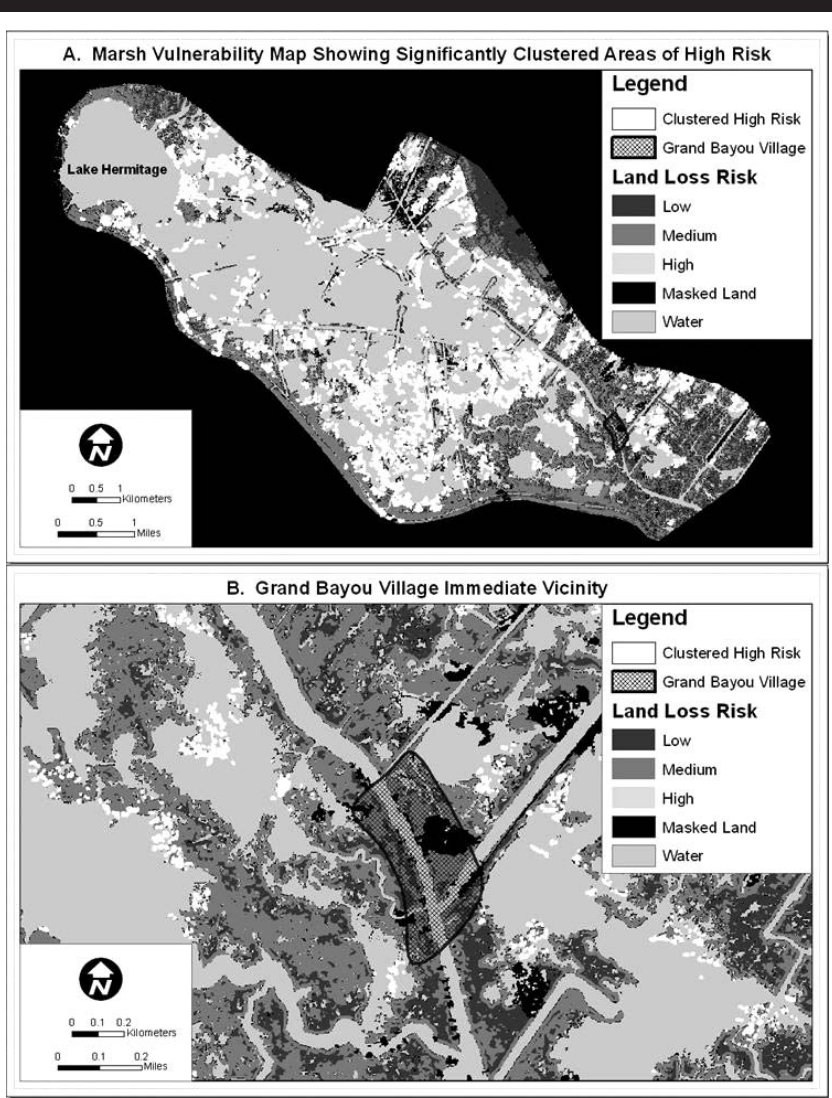

Figure 5. (A) Significantly clustered marsh areas of high risk to loss overlaid on the marsh vulnerability map produced using the results of the biophysical and fragmentation data set analyses. (B) Map showing significantly clustered marsh areas of high risk to loss enlarged to the vicinity of Grand Bayou Village.

knowledge of observed change at these priority areas as it relates to the effect on natural resources and ecosystem health on which the community relies. This GIS analysis allowed the researchers to identify areas of concern, explore different criteria for selection of the most suitable areas for restoration, and rank areas at risk with regard to community concern within a geographical context. The final output consists of a set of digital maps showing optimal areas for strategic coastal restoration activities based on the criteria selected. As an example, the specific criteria for the final map shown in Figure 6 were: (1) areas that corresponded to the most important restoration priority (RP) class; (2) areas that corresponded to both the extreme negative and negative observed change (OC) classes; and (3) areas that were identified as significantly clustered in high risk for loss. When the criteria of the OC input were changed to include only areas of extreme negative observed change, the final map that resulted was modified to show only the clustered high risk of loss areas that met the new criteria. This modification of the input criteria narrowed the output selection areas to the immediate vicinity of Grand Bayou Village. The selected areas were exported to a new data layer so that this information can be used with other maps and data sets. These selected areas

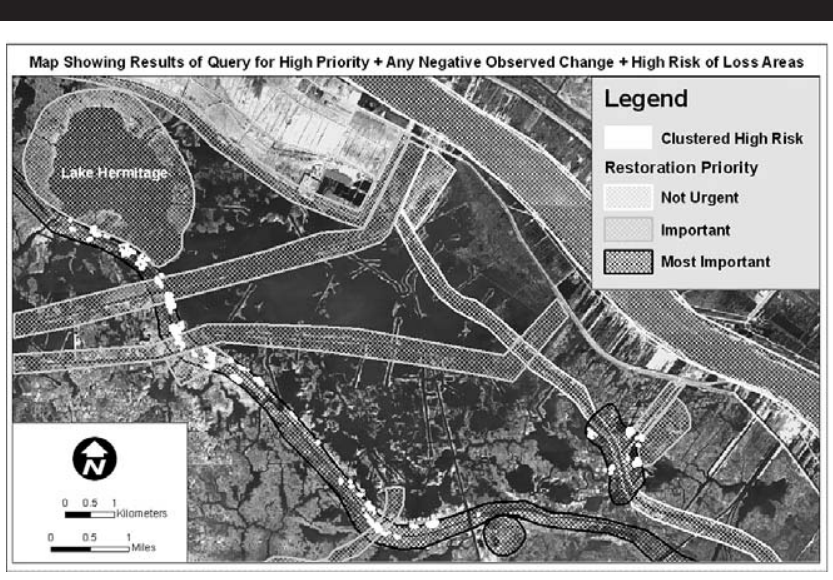

Figure 6. Map result for a query of the integrated GIS showing clustered high risk for loss marsh areas that are within areas of any TEK-based negative OC and the TEK-based most important RP.

identify where there is an urgent need for restoration or other conservation action, and where the greatest restoration benefits for the community can be achieved for a fixed level of available financial resources.

\section{DISCUSSION}

Land loss in the study area was persistent through all time periods observed. There are many driving factors contributing to the land loss detailed in this study, including: naturally occurring subsidence; implementation of the Mississippi River levee system, which halted the river's seasonal overbank flooding that naturally deposited sediment to counteract subsidence; and the construction of a network of oilfield canals into the project area (Boshart and MacInnes, 2000). Also, a major factor of land loss for this area can be attributed to the periodic impacts of storms such as those listed in Table 2, which, according to the related TEK collected, uprooted vegetation and formed areas of broken marsh. The new inlets and expanded waterways allowed increasing tidal exchange and greater salinity fluctuations, which created a stressful habitat for historical vegetation that was less tolerant of these conditions. The degraded vegetation cover further contributed to wetland loss by facilitating erosion (Weller, 1994). Thus, weaker marsh areas eroded more easily with regular tidal action or occasional tropical storm activity.

The majority of the land loss in the study area over the time period studied occurred prior to 1991. The greatest amount of land loss in this area for the time periods studied was observed from 1979 to 1991. Rates of land loss dropped dramatically after 1991 and remained relatively stable in the time periods observed through 2005. The land lost between 2005 and 2009 indicated a slight increase in the trend of land loss observed for the previous three time periods beginning in 1991. These landloss trends are reflected in the graph of proportion of total land area to total water area over the time periods studied (Figure 4). Based on the detailed land-change analysis, it was determined that the study area has undergone a complex morphological evolution that includes large core areas of once- 
unbroken marsh being perforated with small ponds, leading to a significant increase in broken or patchy marsh areas that eventually were lost to open water. This fragmentation and loss pattern was observed throughout the time period investigated.

The land-change assessment methods developed for this study provide the most detailed assessment of landscape evolution to current conditions for this area as a basis for a multi-data set integrated restoration decision-support system. This model provides restoration managers with a means by which to introduce landscape pattern and marsh biophysical parameters associated with plant health into a discussion that is now typically dominated by statistics on the amount of land lost for a given area. The model used to create the marsh vulnerability map can be used to evaluate an area in detail for restoration potential. This methodology demonstrates how scientific field sampling data can be effectively merged within a GIS with information on landscape pattern and trends in order to make marsh vulnerability projections within a spatial context. Furthermore, this methodology is presented as a flexible tool within an image-processing/GIS environment that allows for biophysical and landscape fragmentation variables to be adapted or replaced according to a particular user's needs and the availability of data sets related to these two variables.

Community-based indices that are calculated from numerically coded TEK themes provide a data set that can be used to create a GIS to map locations and features and to represent their associated attributes as derived from TEK. This TEK mapping method allows for a straightforward verification by the informants of the ways in which the TEK was used in this study in a format that facilitates easy assessment. The map format is also conducive to inclusion and integration of TEK into the restoration decision-making process. Currently, TEK is not utilized in restoration planning or adaptive management of current restoration projects. This is due, in part, to a lack of understanding on the part of restoration scientists and engineers of ways in which qualitative TEK data sets can be integrated with quantitative data to support restoration decision making. The coding methods and TEK mapping products produced in this study provide a repeatable solution for incorporating the wealth of local knowledge with scientific data sets, including the historical and projected land-loss maps derived with geospatial technologies that are currently used by restoration managers and scientists.

To ensure acceptance of the TEK/scientific data integration methods produced in this study, the researchers attempted to address typical scientific concerns while incorporating TEK. First, this research ensured that the TEK information obtained for this study involved repeated observations of field sites by several different individual informants to reduce the impact of any information bias. As a result, the information obtained from any one informant did not dominate the "coded" information that was mapped and integrated with the scientific data collected.

Second, TEK data verification and validation procedures were included in the data-collection and integration methods developed for this study. These procedures included meeting with the informants interviewed to review recorded and summarized information from which subsequent inferences were drawn regarding project questions. This was done to ensure that the TEK was recorded and interpreted accurately during the interviews. Verification and validation exercises also served as opportunities for the research team to show the community informants the scientific data and TEK collected, thus providing transparency in our efforts.

Third, although the methods developed in this study focus on blending the TEK of the Grand Bayou community informants with scientific data sets, these methods are generally "repeatable," or applicable, to any proposed or ongoing restoration project that impacts a community where the population has a long history of being intricately tied to the surrounding ecosystem. This situation is the case with many of Louisiana's coastal fishing villages, as well as fishing communities throughout the Gulf Coast. Thus, the integration methodology, as proposed, is not specific to Grand Bayou and can be applied to other areas with similar issues.

Last, in working with the residents as collaborators on this project, they have become more familiar with the capabilities and limitations of remotely sensed imagery, and thus are able to make more informed recommendations based on this technology. Every time the researchers visited Grand Bayou, maps generated from remotely sensed imagery were left with the residents, and the researchers made an effort to explain how the maps were produced. The researchers have also fostered a relationship between community residents and the collaborating OCPR scientists associated with monitoring the study area. This relationship was nonexistent prior to this study.

\section{Sources of Possible Error}

There are small areas of land gain in the land-change maps throughout the time frame studied. This land gain is most likely due to sediment reworking during storm events and the deposition of wrack material, which fills up small ponds and previously shallow submerged areas. There could also be some classification errors due to the presence of aquatic vegetation, such as water hyacinth, as land; however, all images used were acquired during fall through early spring to minimize the presence of floating aquatic vegetation that would have caused confusion in land/water discrimination (Barras et al., 2003). There are also errors with land-water classifications resulting from sun glint and image data-quality issues, which likely contribute to some misclassification of land gain.

The chlorophyll meter and LAI measurements were collected late in the growing season (late October to early November) at a time when natural senescence was occurring in the marsh vegetation. This time frame was not ideal for data collection related to marsh health, given that vegetative stress measured could be due to natural plant phenology in this area. For reasons beyond the control of the research team, image data acquisition was delayed until October 30, 2009, necessitating the delay of concurrent field vegetation measurements.

The main weaknesses of the approach to modeling marsh vulnerability suggested in this research include: the difficulty of separating the correlation between marsh vulnerability and the parameters tested from causality of loss; the difficulty of determining the direction of causality; and the limitations inherent in integrating only chlorophyll content, LAI, and 
landscape fragmentation characteristics into a model for marsh vulnerability, while ignoring other variables that possibly play key roles in the land-loss process. This analysis therefore only provides a prediction of vulnerability from three specific input data sets related to marsh vegetative health, spatial orientation, and proximity to water, an approach which should be complemented by other relevant data sets before a comprehensive understanding of marsh vulnerability to loss for this area can be achieved. Other data sets currently collected and used in the restoration decision-making process that could prove useful for inclusion into this model include hydrologic information related to salinity, water level, turbidity, etc. However, the model developed in this analysis allows for the inclusion of additional variables representing key influences in land change in future marsh vulnerability analyses.

The TEK-based indices were created in order to increase accessibility of this TEK data to restoration decision makers. Following input from collaborating OCPR scientists, it was determined that a visual representation of observed land-loss and priority restoration areas would be more likely utilized in the current restoration process than similar information in text format. The researchers were privileged to witness elaborate, multidimensional storytelling, much of which comprised the TEK collection. We do not propose to represent this traditional practice of storytelling or encompass the complete wealth of TEK collected within these maps, but rather to bridge the gap between the restoration decision-making process and TEK by representing two important community-based attributes (observed change and restoration priority) about features and locations that emerged from the TEK.

\section{Suggestions for Future Research}

The development of the methods and GIS tools implemented in this study and the improved understanding of the ways in which TEK can be merged with scientific data sets to inform restoration decision making illustrate that numerous unanswered questions regarding benefit and cost aspects of knowledge integration and application for coastal restoration exist. Moreover, in order to fully test and further develop the methods and mapping products presented in this study, the results should be applied to other vulnerable coastal ecosystems that serve as livelihood bases for coastal communities. The following is a summary of proposed future directions that might expand upon the findings of this study:

(1) Continuing detailed monitoring of the morphology of the Grand Bayou area is needed in order to determine effectiveness and impacts of the current and proposed restoration projects on the ecosystem, to better understand long-term versus short-term (i.e., episodic storm related) land-loss trends, and to document changes for comparison to projected vulnerability identified in this research.

(2) Possible major causes of land loss identified in this study should be monitored, including oil canals, through subsequent research focusing on future data collection and analyses in an effort to quantify their effects.

(3) Incorporation of economic analyses is needed to deter- mine the cost/benefit ratio associated with incorporating this type of integrated data set into the current process as a means of justifying the additional time and cost, which may preclude it from being utilized more frequently by restoration scientists. If supportive results can be achieved, there will be a compelling precedent for scientists to view more favorably utilizing such a method.

(4) New data sets of the marsh biophysical parameters from field data acquired during the peak of the growing season should be integrated, and then the marsh biophysical estimated mapping process should be repeated to include the new data sets. This will allow for comparison with the biophysical mapping results of this study and may improve the assessment of relative marsh health for inclusion into a revised vulnerability assessment.

(5) New remotely sensed data products with greater spectral resolution should be integrated into the mapping processes described in this study in an attempt to improve correlation with field data. Additional research may be facilitated by better availability and reduced cost of finerresolution remotely sensed satellite data as more remotesensing data options become available to researchers.

\section{CONGLUSIONS}

A review of the current restoration decision-support process shows that the methodologies and GIS tools produced and used in this study are suitable for inclusion into the project prioritization process utilized by OCPR. In addition, the data products generated can inform the process and influence the results with meaningful, new information that is geared toward meeting localized needs rather than regionally based criteria. This study demonstrates that once hot spots of land loss are identified on a regional scale and further prioritization is needed for selection of restoration projects on a local level, the information made available through the methodologies and tools used in this study can be included with other criteria, such as cost estimates, to make more informed restoration decisions.

Land loss in the vicinity of Grand Bayou, and throughout coastal Louisiana, results from a complicated set of environmental and anthropogenic causes, which include canal dredging; subsidence; erosion; storms; levees; and even climate change (Bernier, Morton, and Barras, 2006; Michot, Wells, and Kemmerer, 2004; Penland et al., 1996; Turner, 1997; Walker et al., 1987). Because of its complexity, isolating specific causes and trends of land loss in the study area is a daunting task given limited funding and resources. However, an advantage of working closely with local residents and utilizing TEK is that the study focus can be effectively narrowed to identify the likely major causes of local land loss and to shape the investigation accordingly. In analyzing the TEK obtained for this study, an emphasis was placed by the community informants on the effects of oil company-constructed canals, which dominate the study area today. Secondary ecological effects that resulted from the construction of these canals are noted in the TEK as allowing saltwater intrusion and natural hydrological flow alteration, which in turn accelerated the land loss observed. Furthermore, the TEK analyses identified a time line for 
observed relative land-loss change (i.e., acceleration) and construction of the canals, allowing researchers to identify and acquire image data sets from relevant time periods for use in the scientific assessment of land loss.

Castillo et al. (2005) states that the "generators of scientific knowledge must work closely with its users to identify problems, construct ad hoc solutions, and participate in decision-making processes ranging from the local level to that of policy formulation" ( $p$. 745) so that the results of such research can be "turning scientific findings into actions" (p. 745). The methodology developed to produce the TEK-based maps for this study demonstrates a means by which such qualitative information can be converted into mapping products that are more suitable for inclusion into the existing restoration decision-support system. Moreover, the methodology used to gain access to the TEK utilized in creating the TEKbased maps serves as an example of a way in which scientists can effectively engage local communities as partners in similar collaborative efforts. By seeking a collaborative partnership in assessing impacts and uses, the state officials and the scientists engaged in the restoration analyses also gain support from the commercial and recreational users because the latter are brought in as partners to contribute to the sustainability of the ecosystem on which they depend.

The research team presented the results of this study as an example of the way in which this collaborative partnership can benefit the coastal restoration decision-support process to the LCA S \& T Board at its September 15, 2010, meeting. Subsequently, scientists and restoration management officials in attendance requested the integrated mapping products resulting from this work to use in the current CWPPRA project nominations process for restoration planning and prioritization in the study area. The inclusion of these tools into the CWPPRA project nominations process represents a success in achieving a goal of this study. However, the researchers involved with this study hope to continue to increase dialog and discussion between the two groups, ecosystem users and scientists/ government officials, fostering mutual respect and knowledge transfer that will be sustained beyond the term of this study. If this goal is achieved, the Grand Bayou residents will continue to provide OCPR with ecological insight, informed suggestions, and critique, thus aiding the mapping process, as well as image data set interpretation, and ultimately helping to inform the West Pointe a la Hache restoration decision-making process for the foreseeable future. In doing so, this effort will address the general lack of understanding by physical scientists of the information value that TEK offers, as well as start to bridge the communication gap that typically exists between scientists and traditional knowledge holders as the ecosystem is altered through restoration projects.

\section{ACKNOWLEDGMENTS}

This research is the result of a collaborative effort with the participating residents of the Grand Bayou, Louisiana, community. A huge debt of gratitude is extended to these very gracious and generous people for contributing their knowledge and time to this study. This work has been supported with funding provided by Louisiana State Uni- versity's Coastal Restoration and Enhancement through Science and Technology Program (CREST), and The National Oceanic and Atmospheric Administration's Gulf of Mexico Coastal Storms Program under NOAA Grant NA07OAR4170510 (NOAA), and the Mississippi-Alabama Sea Grant Consortium (MASGC). The funding support of CREST, NOAA, and MASGC is gratefully acknowledged.

\section{LITERATURE CITED}

Anderson, J.R.; Hardy, E.E.; Roach, J.T., and Witner, R.E., 1976. A Land Use and Land Cover Classification System for Use with Remote Sensor Data. U.S. Geological Survey Professional Paper 964, 28p.

Balram, S.; Dragicevic, S., and Meredith, T., 2004. A collaborative GIS method for integrating local and technical knowledge in establishing biodiversity conservation priorities. Biodiversity and Conservation, 13, 1195-1208.

Barras, J.A.; Bernier, J.C., and Morton, R.A., 2008. Land Area Change in Coastal Louisiana-A Multidecadal Perspective (from 1956 to 2006). U.S. Geological Survey Scientific Investigations Map 3019 , scale 1:250,000, 14p.

Barras, J.A.; Beville, S.; Britsch, D.; Hartley, S.; Hawes, S.; Johnston, J.; Kemp, P.; Kinler, Q.; Martucci, A.; Porthouse, J.; Reed, D.; Roy, K.; Sapkota, S., and Suhayda, J., 2003. Historical and Projected Coastal Louisiana Land Changes: 1978-2050. U.S. Geological Survey Open-File Report 03-334, 39p.

Berkes, F.; Colding, J., and Folke, C., 2000. Rediscovery of traditional ecological knowledge as adaptive management. Ecological Applications, 10(5), 1251-1262.

Bernier, J.C.; Morton, R.A., and Barras, J.A., 2006. Constraining rates and trends of historical wetland loss, Mississippi River delta plain, south-central Louisiana. In: Xu, Y.J. and Singh, V.P. (Eds.), Coastal Environment and Water Quality. Highlands Ranch, Colorado: Water Resources Publications, LLC, pp. 371-382.

Boshart, W.M. and MacInnes, A.D., 2000. West Pointe a la Hache Freshwater Diversion BA-04: Progress Report 3 for the Period May 1995 to December 1998, May 2000. Baton Rouge, Louisiana: Louisiana Office of Coastal Protection and Restoration, 29p.

Britsch, L.D. and Dunbar, J.B., 1993. Land loss rates: Louisiana coastal plain. Journal of Coastal Research, 9, 324-338.

Calamia, M.A., 1999. A methodology for incorporating traditional ecological knowledge with geographic information systems for marine resource management in the Pacific. SPC Traditional Marine Resource Management and Knowledge Information Bulletin, 10, 2-12.

Carter, G.A. and Spiering, B.A., 2002. Optical properties of intact leaves for estimating chlorophyll concentration. Journal of Environmental Quality, 31, 1424-1432.

Castillo, A.; Torres, A.; Velazquez, A., and Bocco, G., 2005. The use of ecological science by rural producers: a case study in Mexico. Ecological Applications, 15(2), 745-756.

Cody, R.P. and Smith, J.K., 1991. Applied Statistics and the SAS Programming Language, $4^{\text {th }}$ edition. Upper Saddle River, New Jersey: Prentice Hall, 445p.

Congalton, R. and Green, K., 2009. Assessing the Accuracy of Remotely Sensed Data: Principles and Practices, $2^{\text {nd }}$ edition. Boca Raton, Florida: Taylor \& Francis Group, LLC, 177p.

Coastal Protection and Restoration Authority (CPRA), 2009. Fiscal Year 2010 Annual Plan: Integrated Ecosystem Restoration and Hurricane Protection in Coastal Louisiana, April 27, 2009. Baton Rouge, Louisiana: Coastal Protection and Restoration Authority of Louisiana, 27p.

Crabtree B.F. and Miller, W.L., 1992. Doing Qualitative Research. Newberry Park, California: Sage.

Coastal Wetlands Planning Protection and Restoration Act (CWPPRA), 2006. Wetland Value Assessment Methodology Introduction (prepared by: Environmental Work Group). U.S. Fish and Wildlife Service. http://www.lacoast.gov/reports/wva/ WVA\%20Introduction.pdf (accessed April 1, 2010). 
Dunbar, J.B.; Britsch, L.D., and Kemp, E.B., 1992. Land Loss Rates, Report 3, Louisiana Coastal Plain. U.S. Army Engineers District, New Orleans, Technical Report GL-90-2, 28p.

ESRI (Environmental Systems Research Institute), 2010. ArcGIS Geoprocessing Resource Centers, Landscape Fragmentation Tool help documentation. http://resources.esri.com/geoprocessing/index. $\mathrm{cfm} ? \mathrm{fa}=$ codeGalleryDetails\&scriptID $=16793$ (accessed April 1, 2010).

Ferguson, M.A. and Messier, F., 1997. Collection and analysis of traditional ecological knowledge about a population of Arctic tundra caribou. Arctic, 50(1), 17-28.

Folse, T.M.; West, J.L.; Hymel, M.K.; Troutman, J.P.; Sharp, L.; Weifenbach, D.; McGinnis, T., and Rodrigue, L.B., 2008. A Standard Operating Procedures Manual for the Coast-wide Reference Monitoring System-Wetlands: Methods for Site Establishment, Data Collection, and Quality Assurance/Quality Control. Baton Rouge, Louisiana: Louisiana Coastal Protection and Restoration Authority, Office of Coastal Protection and Restoration, 191p.

Freudenburg, W.R.; Gramling, R.; Laska, S., and Erikson, K.T., 2009. Catastrophe in the Making: The Engineering of Katrina and the Disasters of Tomorrow. Washington, DC: Island Press, 170p.

Gergel, S.E. and Turner, M.G., 2003. Learning Landscape Ecology: A Practical Guide to Concepts and Techniques. New York: Springer-Verlag, $316 \mathrm{p}$.

Gitelson, A.A., 2004. Wide dynamic range vegetation index for remote quantification of biophysical characteristics of vegetation. Journal of Plant Physiology, 161, 165-173.

Gramling, R. and Hagelman, R., 2005. A working coast: people in the Louisiana wetlands. Journal of Coastal Research, Special Issue No. 44, pp. 112-133.

Gustafson, E.J., 1998. Quantifying landscape spatial pattern: what is the state of the art? Ecosystems, 1, 143-156.

Hruschka, D.J.; Schwartz, D.; St. John, D.C.; Picone-Decaro, E.; Jenkins, R.A., and Carey J.W., 2004. Reliability in coding openended data: lessons learned from HIV behavioral research. Field Methods, 16(3), 307-331.

Huntington, H.P., 2000. Using traditional ecological knowledge in science: methods and applications. Ecological Applications, 10(5), $1270-1274$.

Jensen, J.R., 2004. Introductory Digital Image Processing, $3^{\text {rd }}$ edition. Upper Saddle River, New Jersey: Prentice Hall, 526p.

Jensen, J.R.; Ramsey, E.W.; Mackey, H.E., Jr.; Christensen, E.J., and Sharitz, R.R., 1987. Inland wetland change detection using aircraft MSS data. Photogrammetric Engineering and Remote Sensing, 53, 521-529.

Kurasaki, K.S., 2000. Intercoder reliability for validating conclusions drawn from open-ended interview data. Field Methods, 12(3), 179194.

LaCoast, 2008. CWPPRA Restoration Status. http://www.lacoast.gov/ reports/rtc/1997/7.htm (accessed April 1, 2010).

LAI-2000, 1992. LAI-2000 Plant Canopy Analyzer Instruction Manual. Lincoln, Nebraska: LI-COR, Inc., 179p.

Louisiana Coastal Area Science and Technology Program (LCA S\&T), 2010. Summary Report of the January 13-15, 2010, Meeting. New Orleans: Louisiana Coastal Area Science and Technology Program, $16 \mathrm{p}$.

Manning, S.C., 2005. Riding out the Risks: An Ethnographic Study of Risk Perceptions in a South Louisiana Bayou Community. Baton Rouge, Louisiana: Louisiana State University and Agricultural and Mechanical College, Department of Environmental Studies, Master of Science thesis, $125 \mathrm{p}$.

McCarthy, T.S.; Franey, N.J.; Ellery, W.N., and Ellery, K., 1993. The use of SPOT imagery in the study of environmental processes of the Okavango Delta, Botswana. South African Journal of Sciences, 89, $432-436$.

Michot, T.C.; Wells, C.J., and Kemmerer, R.S., 2004. Spatial and Temporal Distribution of Coastal Marsh Dieback in Louisiana, 2000-2003, as Determined from Aerial Surveys. Coastal Restoration Division, Louisiana Department of Natural Resources Technical Report for DNR Contract Number 2512-05-03, 60p.
Mitsch, W.J. and Gosselink, J.G., 2007. Wetlands, $4^{\text {th }}$ edition. Hoboken, New Jersey: John Wiley \& Sons, Inc., 600p.

Nilsson, H.E., 1995. Remote sensing and image analysis in plant pathology. Annual Review of Phytopathology, 15, 489-527.

Ozesmi, S.L. and Bauer, M.E., 2002. Satellite remote sensing of wetlands. Wetlands Ecology and Management, 10, 381-402.

Patton, M.Q., 1990. Qualitative Evaluation and Research Methods, 2nd edition. Newbury Park, California: Sage, 531p.

Penland, S.; Mendelssohn, I.; Wayne, L., and Britsch, D., 1996. Natural and Human Causes of Coastal Land Loss in Louisiana. Baton Rouge, Louisiana: Coastal Studies Institute and Wetland Biogeochemistry Institute, Louisiana State University, 25p.

Petch, J.P.; Pauknerova, E., and Heywood, D.I., 1995. A strategy for GIS in nature conservation: the Zdarske Vrchy project. International Institute for Geo-Information Science and Earth Observation (ITC) Journal, 2, 133-142.

Qi, J.; Chehbouni, A.; Huete, A.R., and Kerr, Y.H., 1994. Modified Soil Adjusted Vegetation Index (MSAVI). Remote Sensing of the Environment, 48, 119-126.

Riiters, K.; Wickham, J.; O'Neill, R.; Jones, B., and Smith, E., 2000. Global-scale patterns of forest fragmentation. Conservation Ecolo$g y, 4(2), 3$.

SAS Institute, Inc., 2002. SAS/STAT Users Guide, version 9.2. Cary, North Carolina: SAS Institute Inc.

Spectrum Technologies, Inc., 2008. FieldScout CM1000 Chlorophyll Meter. http://www.specmeters.com/Chlorophyll_Meters/CM_1000_ Chlorophyll_Meter.html (accessed April 1, 2010).

Steyer, G.D.; Raynie, R.C.; Steller, D.L.; Fuller, D., and Swenson, E., 2000. Quality Management Plan for Coastal Wetlands Planning, Protection, and Restoration Act-Monitoring Program. Louisiana Department of Natural Resources, Coastal Restoration Division Open-File Report 95-01, 112p.

Turner, M.G. and Gardner, R.H., 1991. Quantitative Methods in landscape ecology. New York: Springer-Verlag, 527p.

Turner, M.G.; Gardner, R.H., and O'Neill, R.V., 2003. Landscape Ecology in Theory and Practice: Pattern and Process. New York: Springer-Verlag, 401p.

Turner, R.E., 1997. Wetland loss in the northern Gulf of Mexico: multiple working hypotheses. Estuaries, 20(1), 1-13.

Vigier, B.J.; Pattey, E., and Strachan, I.B., 2004. Narrowband vegetation indexes and detection of disease damage in soybeans. IEEE Geoscience and Remote Sensing Letters, 1(4), 255-259.

Vogt, P.; Ferrari, J.R.; Lookingbill, T.R.; Gardner, R.H.; Riiters, K.H., and Ostapowicz, K., 2009. Mapping functional connectivity. Ecological Indicators, 9, 64-71.

Vogt, P.; Riiters, K.; Estrenguil, C.; Kozak, J.; Wade, T., and Wickham, J., 2007. Mapping spatial patterns with morphological image processing. Landscape Ecology, 22, 171-177.

Walker, J.H.; Coleman, J.M.; Roberts, H.H., and Tye, R.S., 1987. Wetland loss in Louisiana. Geografiska Annaler, Series A, Physical Geography, 69(1), 189-200.

Wang, Y.; Woodstock, C.E.; Buermann, W.; Stenberg, P.; Voipio, P.; Smolander, H.; Hame, T.; Tian, Y.; Hu, J.; Knyazikhin, Y., and Myneni, R.B., 2004. Evaluation of the MODIS LAI algorithm at a coniferous forest site in Finland. Remote Sensing of the Environment, 91, 114-127.

Weller, M.W. (ed.), 1994. Freshwater Marshes: Ecology and Wildlife Management, Wildlife Habitats, Volume 1, 3rd edition. Minneapolis, Minnesota: University of Minnesota Press, 151p.

West, J.; Peterson, K.; Alcina, M., and Laska, S., 2008. Principles of participatory action research (PAR), issues of entrée, and internal review board (IRB) challenges in community/agency/university collaborations. Journal for Community Engaged Research and Learning Partnerships, 1(1), 13-26.http://www.manifestationjournal. org/ (accessed August 1, 2009).

Zhang, M.; Ustin, S.L.; Rejmankova, E., and Sanderson, E.W., 1997. Monitoring Pacific salt marshes using remote sensing. Journal of Ecological Applications, 7(3), 1039-1053. 